31. The MWNT E values were calculated with the assumption that the nanotubes are solid cylinders. TEM imaging of our MWNTs showed that they have hollow cores with diameters $\leq 2 \mathrm{~nm}$. Because the moment of inertia scales as $r^{4}$, this assumption produces only a very small error on the order of $0.01 \%$.

32. O. L. Blakslee, D. G. Proctor, E. J. Seldin, G. B. Spence, T. Weng, J. Appl. Phys. 41, 3373 (1970)

33. D. H. Robertson, D. W. Brenner, J. W. Mintmire Phys. Rev. B 45, 12592 (1992).

34. G. Overney, W. Zhong, D. Tomanek, Z. Phys. D 27 93 (1993)

35. R. S. Ruoff and D. C. Lorentz, Carbon 33, 925 (1995)

36. J. P. Lu, Phys. Rev. Lett. 79, 1297 (1997).

37. The discontinuity in $F-d$ is not due to a discontinuity in the topography. First, high-resolution images demonstrate that the $\mathrm{MoS}_{2}$ substrate is atomically flat in the region where the force discontinuity is observed. Furthermore, the topographic signal, which was recorded at the same time as $F$, is constant across the region of force discontinuity.

38. Mechanical deformation of metal whiskers ultimately leads to a decrease in the initial linear (elastic) F-d behavior. This decrease is, however, due to plastic deformations (4).

39. D. Hull and T. W. Clyne, An Introduction to Composite Materials (Cambridge Univ. Press, Cambridge, 1996).

40. The bending strength is relevant to composites formed with randomly oriented nanotubes or NRs. In a composite made with oriented nanotubes or
NRs, the tensile and compressive strengths should also be considered. The tensile and bending strengths are comparable for $\mathrm{SiC}$ whiskers $(6,7)$ and are also expected to be similar for $\mathrm{SiC}$ NRs. The tensile strength of a carbon nanotube is, however, expected to be substantially larger than the nanotube bending strength and the strength of SiC NRs.

41. We thank J. W. Hutchinson and F. Spaepen for helpful discussions and S. Shepard for assistance with SiO deposition. C.M.L. acknowledges partial support of this work by the NSF Division of Materials Research and the Air Force Office of Scientific Research.

13 June 1997; accepted 19 August 1997

\title{
Enhancement of Protein Crystal Nucleation by Critical Density Fluctuations
}

\author{
Pieter Rein ten Wolde and Daan Frenkel ${ }^{\star}$
}

\begin{abstract}
Numerical simulations of homogeneous crystal nucleation with a model for globular proteins with short-range attractive interactions showed that the presence of a metastable fluid-fluid critical point drastically changes the pathway for the formation of a crystal nucleus. Close to this critical point, the free-energy barrier for crystal nucleation is strongly reduced and hence, the crystal nucleation rate increases by many orders of magnitude. Because the location of the metastable critical point can be controlled by changing the composition of the solvent, the present work suggests a systematic approach to promote protein crystallization.
\end{abstract}

As a result of rapid developments in biotechnology, there has been an explosive growth in the number of proteins that can be isolated. However, the determination of the three-dimensional structures of proteins by $\mathrm{x}$-ray crystallography remains a timeconsuming process. One bottleneck is the difficulty of growing protein crystals good enough for analysis. In his book on this subject, McPherson wrote, "The problem of crystallization is less approachable from a classical analytical standpoint, contains a substantial component of trial and error, and draws more from the collective experience of the past century. ... It is much like prospecting for gold" (1, p. 6). The experiments clearly indicate that the success of protein crystallization depends sensitively on the physical conditions of the initial solution $(1,2)$. It is therefore crucial to understand the physical factors that determine whether a given solution is likely to produce good crystals.

Studies have shown that not just the strength but also the range of the interactions between protein molecules is crucial for crystal nucleation. In 1994, George and Wilson (3) demonstrated that the success of crystallization appears to correlate with the

FOM Institute for Atomic and Molecular Physics, Kruislaan 407, 1098 SJ Amsterdam, Netherlands.

*To whom correspondence should be addressed. value of $B_{2}$, the second osmotic virial coefficient of the protein solution.

The second virial coefficient describes the lowest order correction to the van't Hoff law for the osmotic pressure $\Pi$ :

$$
\Pi / \rho k_{B} T=1+B_{2} \rho+\left(\text { terms of order } \rho^{2}\right)
$$

where $\rho$ is the number density of the dissolved molecules, $k_{\mathrm{B}}$ is Boltzmann's constant, and $T$ is the absolute temperature. For macromolecules, $B_{2}$ can be determined from static light-scattering experiments (4). Its value depends on the effective interaction between a pair of macromolecules in solution (5).

George and Wilson measured $B_{2}$ for a number of proteins in various solvents. They found that for those solvent conditions that are known to promote crystallization, $B_{2}$ was restricted to a narrow range of small negative values. For large positive values of $B_{2}$, crystallization did not occur at all, whereas for large negative values of $B_{2}$, protein aggregation rather than crystallization took place.

Rosenbaum, Zamora, and Zukoski $(6,7)$ established a link between the work of George and Wilson and earlier studies of the phase behavior of spherical, uncharged colloids (8-11). Since the theoretical work of Gast, Russel, and Hall (8), it has been known that the range of attraction between spherical colloids has a drastic effect on the appearance of the phase diagram. If the range of attraction is long in comparison with the diameter of the colloids, the phase diagram of the colloidal suspension resembles that of an atomic substance, such as argon. Depending on the temperature and density of the suspension, the colloids can occur in three phases (Fig. 1A): a dilute colloidal fluid (analogous to the vapor phase), a dense colloidal fluid (analogous to the liquid phase), and a colloidal crystal phase. However, when the range of the attraction is reduced, the fluid-fluid critical point moves toward the triple point, where the solid coexists with the dilute and dense fluid phases. If the range of attraction is made even shorter (less than $25 \%$ of the colloid diameter), two stable phases remain, one fluid and one solid (Fig. 1B). However, the fluid-fluid coexistence curve survives in the metastable regime below the fluid-solid coexistence curve (Fig. 1B). This is indeed found in experiments $(11,12)$ and simulations (10). This observation is relevant for solutions of globular proteins, because they often have short-range attractive interactions. A series of studies (13-15) showed that the phase diagram of a variety of proteins is of the kind shown in Fig. 1B. Moreover, the range of the effective interactions between proteins can be changed by the addition of nonadsorbing polymer (such as polyethylene glycol) $(11,16)$ or by changing the $\mathrm{pH}$ or salt concentration of the solvent $(1,2)$.

Rosenbaum, Zamora, and Zukoski $(6,7)$ observed that the conditions under which a large number of globular proteins can be made to crystallize map onto a narrow temperature range, or more precisely, a narrow range in the value of the osmotic second virial coefficient of the computed fluid-solid coexistence curve of colloids with short-range attraction (10). Several authors had already noted that a similar crystallization window exists for colloidal suspensions (17). Here our aim was to use simulation to gain insight into the physical mechanism responsible for the en- 
hanced crystal nucleation. We found that the presence of a metastable fluid-fluid critical point is essential.

The rate-limiting step in crystal nucleation is the crossing of a free-energy barrier. In our simulations we computed the barrier for homogeneous crystal nucleation for a model globular protein. Our model system has some of the essential features needed to get a proteinlike phase diagram of the type shown in Fig. 1B - the particles repel strongly at short distances and attract at slightly larger distances.

For the interaction between the particles, we chose a suitable generalization of the Lennard-Jones potential:

$v(r)$

$=\frac{4 \varepsilon}{\alpha^{2}}\left\{\frac{1}{\left[\left(\frac{r}{\sigma}\right)^{2}-1\right]^{6}}-\alpha \frac{1}{\left[\left(\frac{r}{\sigma}\right)^{2}-1\right]^{3}}\right\}$

where $\sigma$ denotes the hard-core diameter of the particles, $r$ is the interparticle distance, and $\varepsilon$ is the depth of the potential well. The potential in Eq. 2 provides a simplified description of the effective interaction between real proteins in solution: It accounts both for direct and for solvent-induced interactions between the globular proteins. The width of the attractive well can be adjusted by varying the parameter $\alpha$. We found that for $\alpha \approx 50$, the system reproduced the phase behavior of protein solutions studied in (13-15), that is, the fluidfluid coexistence curve was located in the metastable region $\sim 20 \%$ below the equilibrium crystallization curve.

Conventional molecular dynamics simulations cannot be used to study crystal nucleation under realistic conditions. The reason is that the formation of a critical nucleus is a rare event; crystallization in real protein solutions may take days or weeks. In a simulation, the situation would be worse because the volume that can be studied by simulation is orders of magnitude smaller, and the probability of crystal formation is decreased by the same amount. Moreover, the computational cost of molecular dynamics simulations that cover more than $10^{-8} \mathrm{~s}$ becomes prohibitive. Hence, the problem has to be approached in a different way. In the present work, we used an approach (18) based on the Bennett-Chandler numerical scheme to compute the rate of activated processes (19). The rate $\Gamma$ can be written as the product of two factors:

$$
\Gamma=\exp \left(-\Delta \mathrm{G}^{*} / k_{\mathrm{B}} T\right) \times v
$$

where $\Delta \mathrm{G}^{*}$ is the height of the (Gibbs) free-energy barrier separating the metastable fluid from the crystal phase. The factor $\exp \left(-\Delta G^{*} / k_{\mathrm{B}} T\right)$ denotes the probability that a spontaneous fluctuation will result in
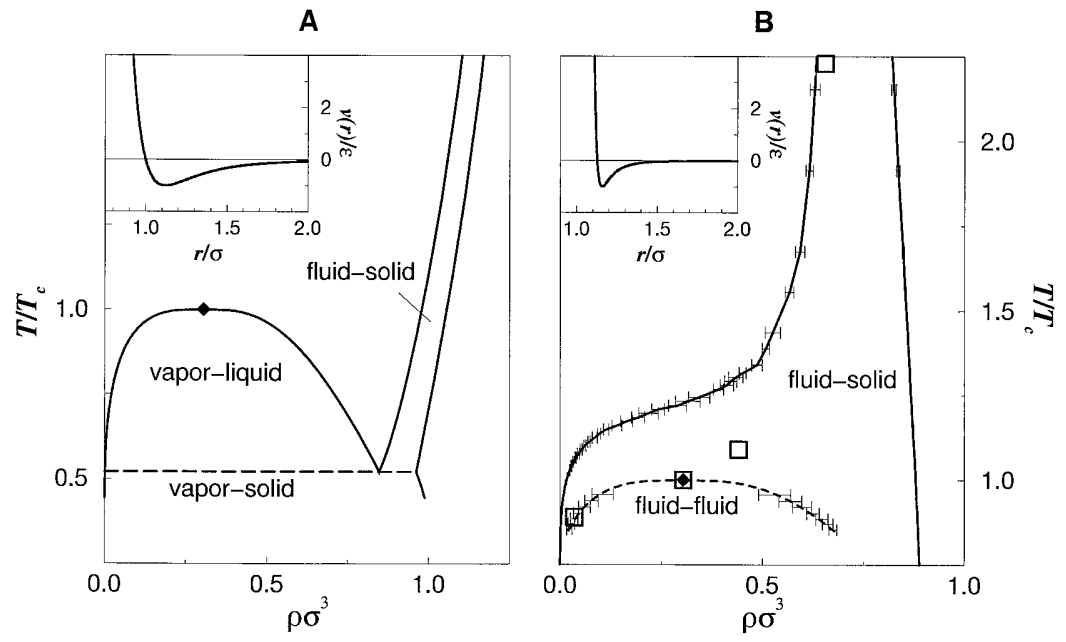

Fig. 1. (A) Typical phase diagram of a molecular substance with a relatively long-range attractive interaction. This phase diagram corresponds to the Lennard-Jones 6-12 potential $\left\{v(r)=4 \varepsilon\left[(\sigma / r)^{12}-\right.\right.$ $\left.(\sigma / r)^{6}\right]$, solid curve in insert\} (24). The dashed line indicates the triple point. (B) Typical phase diagram of colloids with short-range attraction. The phase diagram was computed for the potential given in Eq. 2 (solid curve in insert), with $\alpha=50$. In both figures, the temperature is in units of the critical temperature $T_{\mathrm{C}}$, and the number density is in units $\sigma^{-3}$, where $\sigma$, the effective diameter of the particles, is defined in the expression for $v(r)$. The diamonds indicate the fluid-fluid critical points. In (A) and (B) the solid lines indicate the equilibrium coexistence curves. The dashed curve in (B) indicates the metastable fluid-fluid coexistence. Crystal-nucleation barriers were computed for the points denoted by open squares.

the formation of a critical nucleus; it depends strongly on the degree of supercooling. The kinetic prefactor $v$ is a measure of the rate at which critical nuclei, once formed, transform into larger crystallites. It depends only weakly on supercooling, unless the system is close to gelation (11). Below, we discuss simulations under conditions away from the gelation curve. Hence, the variation of the nucleation rate is dominated by the variation in the barrier height $\Delta \mathrm{G}^{*}$. A rough estimate of $\Delta \mathrm{G}^{*}$ is given by classical nucleation theory (20),

$$
\Delta \mathrm{G}^{*} / k_{\mathrm{B}} T=16 \pi \gamma^{3} /\left(3 k_{\mathrm{B}} T \rho^{2} \Delta \mu^{2}\right)
$$

where $\gamma$ is the free-energy density per unit area of the solid-fluid interface, $\rho$ is the number density of the solid phase, and $\Delta \mu$ is the difference in chemical potential between the fluid and the solid. It is the thermodynamic driving force for crystallization.

To compute the free-energy barrier that the system must overcome to form a critical crystal nucleus, we used the umbrella-sampling Monte Carlo scheme of Valleau (18, 21). This is a biased Monte Carlo scheme that makes it possible to concentrate the sampling in the region of the free-energy barrier. Without the bias, this region of configuration space is effectively inaccessible. To compute the free energy as a function of the size of the incipient crystal nucleus, we must be able to identify which particles belong to the crystal nucleus. As discussed in (18), each particle can be classified as either solidlike or liquidlike by analyzing the local symmetry of its sur- roundings. If the distance between two solidlike particles is less than $1.5 \sigma$, they are considered to be connected and belong to the same cluster. We denote the number of solidlike particles belonging to a given crystal nucleus by $N_{\text {crys }}$. Crystallization near the metastable fluid-fluid critical point is strongly influenced by the large density fluctuations that occur in the vicinity of such a critical point; therefore, the freeenergy barrier associated with formation of dense, liquidlike droplets should also be considered. Hence, for every particle, we determined not just the structure of its surroundings but also the local density. In the same way, the size of a high-density cluster (be it solid or liquidlike) can be defined as the number of connected particles, $N_{\rho}$, that have a significantly denser local environment than particles in the remainder of the system. In our simulations, the free-energy "landscape" of the system was determined as a function of the two coordinates $N_{\text {crys }}$ and $N_{\rho}$. In a crystal nucleation event, the beginning is from the homogeneous liquid $\left(N_{\text {crys }} \approx N_{\rho} \approx 0\right)$. The free energy then increases until it reaches a saddle-point (the critical nucleus). From there on, the crystal will grow spontaneously.

We first computed the phase diagram shown in Fig. 1B using the techniques described in (10) and then studied the freeenergy barrier for crystal nucleation at four different points in the phase diagram: one well above the metastable critical point $\left(T=2.23 T_{c}\right)$, one at $T_{c}$, and the remaining two at $0.89 T_{c}$ and $1.09 T_{c}$. The degree 
Fig. 2. Contour plots of the free-energy landscape along the path from the metastable fluid to the critical crystal nucleus for our system of spherical particles with short-range attraction. The curves of constant free energy are drawn as a function of $N_{\rho}$ and $N_{\text {crys }}$ and are separated by $5 k_{\mathrm{B}} T$. (A) The free-energy landscape well below the critical temperature $\left(T / T_{\mathrm{c}}=0.89\right)$. The lowest free-energy path to the critical nucleus is indicated by a dashed curve. This curve corresponds to the formation and growth of a highly crystalline cluster. (B) As (A),
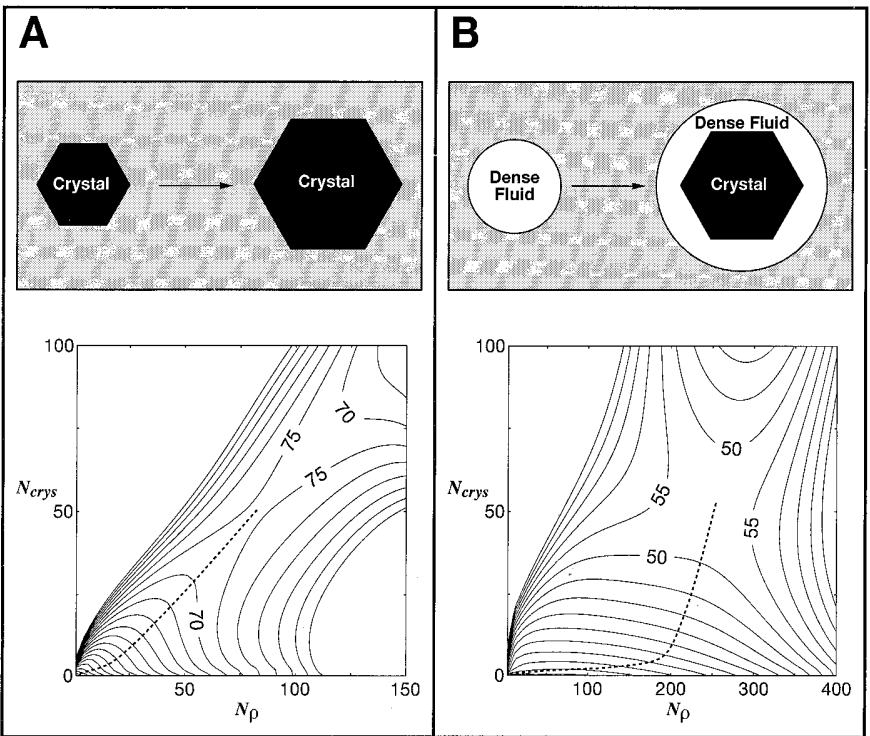
but for $T=T_{\mathrm{c}}$. In this case, the free-energy valley (dashed curve) first runs parallel to the $N_{\rho}$ axis (formation of a liquidlike droplet), and then moves toward a structure with a higher crystallinity (crystallite embedded in a liquidlike droplet). The free-energy barrier for this route is much lower than the one in (A).

of supercooling was chosen such that classical nucleation theory would predict the same value of $\Delta \mathrm{G}^{*} /\left(k_{\mathrm{B}} T\right)$ for all systems. To estimate $\Delta \mathrm{G}^{*}$ from Eq. 4 , we used

$$
\Delta \mu \approx \Delta H\left(T_{\mathrm{m}}-T\right) / T_{\mathrm{m}}
$$

where $\Delta H$ is the enthalpy of melting at the coexistence temperature $T_{\mathrm{m}}$. Turnbull's empirical rule was used to estimate the surface free-energy $\gamma$, which states that $\gamma$ is proportional to $\Delta H(20)$. For all points, we studied the free-energy landscape and the lowest free-energy path to the critical nucleus.

We found that away from $T_{c}$ (above and below), the path of lowest free energy is one where the increase in $N_{\rho}$ is proportional to the increase in $N_{\text {crys }}$ (Fig. 2A). Such behavior is expected if the incipient nucleus is a small crystallite. However, around $T_{c}$, critical density fluctuations lead to a striking change in the free-energy landscape (Fig. 2B). First, the route to the critical nucleus leads through a region where $N_{\rho}$ increases while $N_{\text {crys }}$ is still essentially zero. In other words, the first step toward the critical nucleus is the formation of a liquidlike droplet. Then, beyond a certain critical size, the increase in $N_{\rho}$ is proportional to $N_{\text {crys }}$, that is, a crystalline nucleus forms inside the liquidlike droplet.

Clearly, the presence of large density fluctuations close to a fluid-fluid critical point effects the route to crystal nucleation. But, more importantly, the nucleation barrier close to $T_{c}$ is much lower than at either higher or lower temperatures (Fig. 3). The observed reduction in $\Delta \mathrm{G}^{*}$ near $T_{\mathrm{c}}$ by $\sim 30 k_{\mathrm{B}} T$ corresponds to an increase the nucleation rate by a factor $10^{13}$. One interpre- tation of this observation is that near the fluid-fluid critical point, the wetting of the crystal nucleus by a liquidlike layer results in a value of the interfacial free energy $\gamma$, and therefore of the barrier height $\Delta \mathrm{G}^{*}$, that is much lower than would be estimated on the basis of Turnbull's rule. In fact, Haas and Drenth (22) noted that the experimentally determined interfacial free energy of small protein crystals (23) is much smaller than the value predicted on the basis of their version of Turnbull's rule.

The conventional way to lower the crystal nucleation barrier is to prepare a more supersaturated solution. However, highly supersaturated solutions tend to form aggregates rather than crystals $(3,6,7,11,12$, $15)$. Moreover, in such a solution, the thermodynamic driving force for crystallization $\left(\mu_{\text {liq }}-\mu_{\text {cryst }}\right)$ is also enhanced. As a consequence, the crystallites that nucleate will grow rapidly and far from perfectly (2). One of the implications of our finding that the crystal nucleation barrier is reduced near $I_{c}$ is that one can selectively speed up the rate of crystal nucleation, without increasing the rate of crystal growth, or the rate at which amorphous aggregates form. This can be achieved by adjusting the solvent conditions (for instance, by the addition of nonionic polymer) and thereby changing the range of interaction, such that a metastable fluid-fluid critical point is located just below the sublimation curve.

Our description of the early stages of protein crystallization is highly simplified, yet we suggest that the mechanism for enhanced crystal nucleation described here is quite general. The phase diagram shown in Fig. $1 \mathrm{~B}$ is likely to be the rule

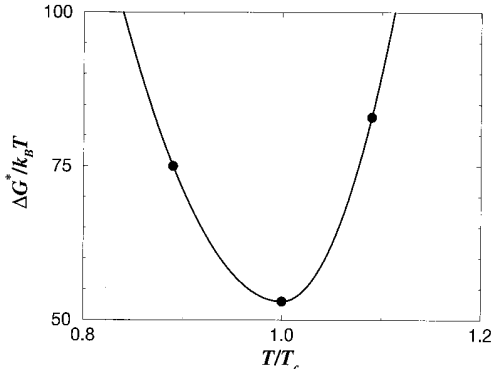

Fig. 3. Variation of the free-energy barrier for homogeneous crystal nucleation, as a function of $T / T_{c}$, in the vicinity of the critical temperature. The solid curve is a guide to the eye. The nucleation barrier at $T=2.23 T_{\mathrm{C}}$ is $128 \mathrm{~K}_{\mathrm{B}} T$ and is not shown in this figure. If Turnbull's phenomenological rule for $\gamma$ would hold (20), Eq. 4 would predict a constant nucleation barrier. But the simulations show that the nucleation barrier goes through a minimum around the metastable critical point.

rather than the exception for compact macromolecules. Moreover, it occurs both in the bulk and in (quasi) two-dimensional systems (such as membranes). It is therefore tempting to speculate that nature already makes extensive use of critical density fluctuations to facilitate the formation of ordered structures.

\section{REFERENCES AND NOTES}

1. A. McPherson, Preparation and Analysis of Protein Crystals (Krieger, Malabar, FL, 1982)

2. S. D. Durbin and G. Feher, Annu. Rev. Phys. Chem. 47, 171 (1996); F. Rosenberger, J. Cryst. Growth 166, 40 (1996)

3. A. George and W. W. Wilson, Acta Crystallogr. D 50, 361 (1994).

4. E. G. Richards, An Introduction to Physical Properties of Large Molecules in Solution (Cambridge Univ. Press, Cambridge, 1980).

5. See, for example, T. L. Hill, An Introduction to Statistical Thermodynamics (Dover, New York, 1986), chap. 15.

6. D. Rosenbaum, P. C. Zamora, C. F. Zukoski, Phys. Rev. Lett. 76, 150 (1996).

7. D. F. Rosenbaum and C. F. Zukoski, J. Cryst. Growth 169, 752 (1996).

8. A. P. Gast, W. B. Russell, C. K. Hall, J. Colloid Interface Sci. 96, 251 (1983); 109, 161 (1986).

9. H. N. W. Lekkerkerker et al., Europhys. Lett. 20, 559 (1992).

10. M. H. J. Hagen and D. Frenkel, J. Chem. Phys. 101 , 4093 (1994)

11. S. M. llett, A. Orrock, W. C. K. Poon, P. N. Pusey, Phys. Rev. E 51, 1344 (1995).

12. W. C. K. Poon, A. D. Pirie, P. N. Pusey, Faraday Discuss. 101, 65 (1995); W. C. K. Poon, Phys. Rev. E 55, 3762 (1997).

13. C. R. Berland et al., Proc. Natl. Acad. Sci. U.S.A. 89 1214 (1992); N. Asherie, A. Lomakin, G. B. Benedek, Phys. Rev. Lett. 77, 4832 (1996).

14. M. L. Broide, T. M. Tominc, M. D. Saxowsky, Phys Rev. E 53, 6325 (1996).

15. M. Muschol and F. Rosenberger, J. Chem. Phys. 107, 1953 (1997).

16. A. McPherson, J. Biol. Chem. 251, 6300 (1976)

17. A. Kose and S. Hachisu, J. Colloid Interface Sci. $\mathbf{5 5}$ 487 (1976); C. Smits et al., Phase Transitions 21 157 (1990).

18. P. R. ten Wolde, M. J. Ruiz-Montero, D. Frenkel, Phys. Rev. Lett. 75, 2714 (1995); J. Chem. Phys. 104, 9932 (1996). 
19. C. H. Bennett, in Diffusion in Solids: Recent Developments, A. S. Nowick and J. J. Burton, Eds. (Academic Press, New York, 1975), pp. 73-113; D. Chandler, J. Chem. Phys. 68, 2959 (1978).

20. See, for example, K. F. Kelton, in Crystal Nucleation in Liquids and Glasses, H. Ehrenreich and D. Turnbull, Eds. (Academic Press, Boston, 1991), vol. 45 pp. 75-177. As shown in this reference, the constant of proportionality is system-specific.

21. G. M. Torrie and J. P. Valleau, Chem. Phys. Lett. 28
578 (1974).

22. C. Haas and J. Drenth, J. Cryst. Growth 154, 126 (1995).

23. A. J. Malkin and A. McPherson, ibid 128, 1232 (1992); ibid. 133, 29 (1993).

24. J. P. Hansen and L. Verlet, Phys. Rev. 184, 151 (1969).

25. We thank E. J. Meijer for computing part of the phase diagram in Fig. 1B, and A. van Blaaderen, M. Dogterom, J. Drenth, J. M. W. Frenken, S. Fraden, C.
Haas, H. N. W. Lekkerkerker, B. Smit, R. Sear, J. T. M. Walraven, and C. Zukoski for critical reading of the manuscript. Supported by Scheikundig Onderzoek Nederland and by Fundamenteel Onderzoek der Materie (FOM) with financial aid from Nederlandse Organisatie voor Wetenschappelijk Onderzoek. Computer time was provided by Nationale Computer Faciliteiten.

9 June 1997; accepted 11 August 1997

\title{
Reversible Tuning of Silver Quantum Dot Monolayers Through the Metal-Insulator Transition
}

\author{
C. P. Collier, R. J. Saykally, J. J. Shiang, S. E. Henrichs, \\ J. R. Heath*
}

The linear and nonlinear $\left(\chi^{(2)}\right)$ optical responses of Langmuir monolayers of organically functionalized silver quantum dots were measured as a continuous function of interparticle separation under near-ambient conditions. As the distance between metal surfaces was decreased from 12 to $\sim 5$ angstroms, both quantum and classical effects were observed in the optical signals. When the separation was less than 5 angstroms, the optical second-harmonic generation (SHG) response exhibited a sharp discontinuity, and the linear reflectance and absorbance began to resemble those of a thin metallic film, indicating that an insulator-to-metal transition occurred. This transition was reversible.

Recent developments in chemical techniques for producing narrow size distributions of various metal (1) and semiconductor (2-4) quantum dots, and for fabricating ordered superlattices from these nanocrystals, have sparked much interest in the possibility of forming solids that have electrical and optical properties that could be tuned through chemical control over particle size, stoichiometry, and interparticle separation (5-10). In principle, control over interparticle separation provides a means for controlling both quantum and classical coupling interactions. This is of practical importance because control of quantum interactions would provide a route to preparing a solid of quantum dot "atoms" and precisely tuning the electronic properties of that solid by controlling the wave function overlap between adjacent particles. Although recent reports have documented classical (energy-transfer) coupling $(11,12)$ between semiconductor quantum dots $(13,14)$, quantum coupling has not been observed. Nor has quantum coupling been documented in coinage metal quantum dots, even though classical coupling [in the form of color changes (15)] and percolation phe-

C. P. Collier and R. J. Saykally, Department of Chemistry, University of California, Berkeley, CA 94720, USA.

J. J. Shiang, S. E. Henrichs, J. R. Heath, Department of Chemistry and Biochemistry, University of California, 405 Hilgard Avenue, Los Angeles, CA 90095, USA.

*To whom correspondence should be addressed. nomena (16) have long been observed in such systems.

Here, we describe in situ measurements of both the linear and nonlinear optical properties of organically functionalized silver nanocrystal Langmuir monolayers as a continuous function of interparticle separation distance. As the monolayer is compressed from an average separation between the surfaces of the metal cores $(\delta)$ of 12 $( \pm 2) \AA$ to $\sim 5( \pm 2) \AA$, the linear and nonlinear optical properties reveal evidence of both classical and quantum interparticle coupling phenomena. Below $\delta \sim 5 \AA$, evidence for a sharp insulator-to-metal transition is observed in both optical signals. The nonlinear optical response abruptly decreases to a nearly constant value, and the linear reflectance drops precipitously until it matches that of a continuous metallic film. This transition is reversible: The particles can be redissolved (as a colloid), or, if the trough barriers are opened, the film is again characterized by the optical properties of near-isolated silver nanocrystals.

Silver quantum dots are expected to exhibit strong linear and nonlinear optical responses (17). The linear response is dominated by the surface plasmon resonance $\omega_{\text {sp }}$. This transition, which has no molecular analog, is characterized by an oscillator strength resembling the physical dimensions of the particle (18). $\omega_{\mathrm{sp}}$ can be modeled as the free (valence) electrons in the particle responding to an applied electromagnetic field, with the positive nuclear cores providing a restoring potential. For particles in the size range considered here ( 2 to $5 \mathrm{~nm}$ ), $\omega_{\mathrm{sp}}$ is determined by the free electron density in the particle and by the dielectric surrounding the particle surface. The resonance width is determined by the time scale for electron scattering at the particle boundaries and is dependent on particle size. The physical picture describing the nonlinear optical response is somewhat different. Consider an electron cloud associated with a nanoparticle. $\chi^{(1)}$ is a measurement of the polarizability of the cloud, which scales as the volume of the particle. $\chi^{(2)}$, the second-order susceptibility, is a measurement of how $\chi^{(1)}$ changes with respect to an applied electric field. A requirement for a nonzero $\chi^{(2)}$ is a lack of inversion symmetry, a condition that is met by a supported monolayer of nanocrystals. $\chi^{(2)}$ is almost exclusively sensitive only to the optical response of the crystallites and is a near background-free measurement.

Both the linear and nonlinear optical responses should provide excellent probes of interparticle coupling. When particles are brought into close proximity to one another, the dielectric surrounding a single particle is strongly modified by the presence of adjacent (conducting) spheres, resulting in shifts (to lower energy) of $\omega_{\mathrm{sp}}$. For quantum mechanical coupling, delocalization of charge carriers over multiple particles increases the scattering lifetime and leads to narrower plasmon linewidths. In the limiting case of complete electron delocalization between particles (that is, the formation of a continuous metal film), strong carrier absorption at energies below $\omega_{\mathrm{sp}}$ and low reflectivity are expected. For example, consider the familiar case of thin metal films, which tend to be darkly colored. Only when a metal film is substantially thicker than the optical skin depth does it become reflective.

$\chi^{(2)}$ should also be an excellent probe of interparticle coupling. A nonlinear optical response is enhanced if the resonant state has both high oscillator strength and large volume (19-21), criteria that are satisfied by the particles discussed here. For classical coupling, local field effects should modify $\chi^{(2)}$ according to multipole coupling models (22). In contrast, quantum mechanical cou- 